\title{
Spinal cord injuries and bowel stomas: timing and satisfaction with stoma formation and alterations in quality of life
}

\author{
F. van Ginkel ${ }^{1}$ M. W. M. Post $\mathbb{D}^{1,2} \cdot$ W. X. M. Faber $\mathbb{D}^{3} \cdot$ V. Meij ${ }^{4} \cdot$ J. M. Stolwijk-Swüste $\mathbb{D}^{1,5}$
}

Received: 19 July 2020 / Revised: 16 December 2020 / Accepted: 27 December 2020

(c) The Author(s), under exclusive licence to International Spinal Cord Society 2021

\begin{abstract}
Study design Cross-sectional study.

Objectives Neurogenic bowel dysfunction (NBD) is frequent among individuals with spinal cord injury (SCI) and is often difficult to treat. A bowel stoma is considered the last-resort treatment option for individuals with SCI and severe NBD. This study aims to explore whether individuals are satisfied with their bowel stoma and how they feel about the timing of stoma formation. Furthermore, we want to explore side effects addressing diversion colitis and changes in quality of life (QOL) after stoma formation.

Setting Netherlands, community.

Methods We included participants aged at least 18 years with a traumatic or non-traumatic SCI and bowel stoma. Questions regarding timing of stoma formation, alterations in QOL after stoma formation and experienced side effects of the bowel stoma were addressed in an online questionnaire.

Results In total 23 participants filled out the online survey. Twenty-two participants (96\%) were satisfied with their bowel stoma and $83 \%$ felt their stoma was placed too late or far too late. The large majority $(>80 \%)$ reported improvements in the four QOL domains after the procedure. Nine participants reported stoma-related problems in the last month. In the last three months, seven participants (30\%) reported to have diversion colitis. Four of these seven participants experienced this at least once a week or more. Two participants stated this had moderate influence on daily activities.

Conclusions Most participants with SCI experienced improvement in QOL and in retrospect wanted their bowel stoma earlier. Early, extensive conversations to inform individuals about bowel stoma as a treatment option is recommended.
\end{abstract}

$\triangle$ J. M. Stolwijk-Swüste

j.stolwijk@dehoogstraat.nl

1 Centre of Excellence for Rehabilitation Medicine, Brain Centre Rudolf Magnus, University Medical Centre Utrecht, Utrecht University and De Hoogstraat Rehabilitation, Utrecht, The Netherlands

2 University of Groningen, University Medical Centre Groningen, Centre for Rehabilitation, Groningen, The Netherlands

3 Department of Spinal Cord Injury, Rehabilitation centre Heliomare, Wijk aan Zee, The Netherlands

4 Department of Gastrointestinal Surgery, University Medical Centre Utrecht, Utrecht, The Netherlands

5 Department of Spinal Cord Injury and Orthopedics, De Hoogstraat Rehabilitation, Utrecht, The Netherlands

\section{Introduction}

One of the most often reported secondary complications in individuals with spinal cord injury (SCI) is neurogenic bowel dysfunction (NBD). NBD can result in fecal incontinence and obstipation, and thereby has a negative influence on a person's quality of life (QOL) [1,2]. Current guidelines refer to a stepped up pyramid tool for bowel management in individuals with SCI [3]. However, these guidelines are based on evidence with low methodological quality [3, 4]. First treatment options include optimizing dietary and fluid adjustments or the use of pharmacologics (e.g., stool softeners, stimulant laxatives, and bulking agents). When these noninvasive methods do not achieve satisfactory bowel management, more invasive techniques can be used (e.g., perianal/rectal stimulation technique, manual removal of feces or transanal irrigation). Eventually, surgical techniques such as the implantation of electrical stimulation systems, antegrade colonic enemas, or the 
formation of a bowel stoma are treatment options for individuals with SCI if fecal incontinence or obstipation still persists. Formation of a bowel stoma is seen as the last resort treatment option because it is invasive and cannot easily be made undone. Individuals with SCI can benefit from a bowel stoma, especially those with good hand function who can take care of their own stoma [5]. Another benefit for individuals with SCI and bowel stoma is the reduced time spent for the evacuation of feces [6]. The formation of a bowel stoma in individuals with $\mathrm{SCI}$ is a safe procedure [7]. However, there is not much literature on the impact of a bowel stoma in regard to self-reported health related QOL for individuals with SCI. This is the same with regard to self-reported satisfaction with a bowel stoma and the moment of getting this bowel stoma. Short and long term complications of stoma formation such as skin complications, parastomal hernias and stomal stenosis have been well described in non-SCI individuals [8]. One of the complications following stoma formation is diversion colitis. Diversion colitis is caused by inflammation of the defunctionalized, neglected part of the colon. This causes (in) voluntary mucus and/or blood discharge, which can have invalidating consequences for individuals [9]. Literature on diversion colitis and its impact on daily life in individuals with SCI and bowel stoma is scarce.

\section{Research question}

This study aims to answer the following research questions:

(1) Are individuals with SCI and bowel stoma satisfied with the decision and the moment of getting this stoma?

(2) Do individuals with SCI experience alterations in QOL after stoma formation?

(3) How many individuals report adverse effects such as diversion colitis?

\section{Materials and methods}

\section{Design}

Cross-sectional survey.

\section{Participants}

The inclusion criteria were as follows: (1) Age of participant at least 18 years; (2) having a traumatic or nontraumatic SCI; and (3) the presence of a bowel stoma. This study was approved by the local Ethics committees of De
Hoogstraat Rehabilitation and Rehabilitation Center Heliomare.

\section{Procedures}

Individuals eligible for the study were identified through De Hoogstraat Rehabilitation, Rehabilitation Center Heliomare, the UMC Utrecht, and Coloplast Netherlands. Coloplast is one of the main suppliers of continence materials for individuals with a bowel stoma in the Netherlands. Participants were contacted with a letter of introduction and an attached link to the online questionnaire. Further, a call for participation was published in the online newsletter of the Dutch spinal cord patient organization and the Dutch organization of bowel stoma patients. Those who were interested to join the study contacted the research team after which they received a personalized link to the online questionnaire. Participants had to confirm their consent for participation to start the questionnaire.

\section{Instruments and definitions}

Questions regarding demographic and disease characteristics were asked. Level and completeness of SCI were obtained by self-report. Participants were asked if they had "tetraplegia" (absent sensory and/or muscle weakness in all limbs) or "paraplegia" (sensory and/or muscle weakness without involvement of the upper limbs). Completeness of SCI was asked for as "complete" (no muscle strength and sensation below the level of the lesion), or 'incomplete' (some muscle strength or sensation below the level of the lesion). Years since SCI was calculated with age since SCI and age of participant. We used the month and year of stoma formation together with the day of replying to the online questionnaire to calculate the years since bowel stoma. Data regarding participants' bowel stoma were obtained by self-report. Type of bowel stoma was asked for as "small intestine bowel stoma" (ileostomy), "large intestine bowel stoma" (colostomy), or "unknown". Type of bowel stoma was asked for as "single looped" or "double looped".

Severity of bowel symptoms was measured with a stomamodified version of the NBD Score, which we named the "Stoma-Modified Neurogenic Bowel Dysfunction (SMNBD) questionnaire" [10]. Modification was required because some of the NBD items are not applicable to individuals with a bowel stoma. Also, to the best of our knowledge, a questionnaire specific to individuals with SCI and a bowel stoma does not exist. Therefore, we adapted the NBD while keeping it as comparable as possible to the original NBD. "Frequency of defecation" was changed to "Frequency of emptying stoma sack"; "Time used for each evacuation" was changed to "Time spending emptying stoma sack"; "Uneasiness, headache or perspiration during 
defecation" was changed to "Uneasiness, headache, or perspiration while emptying stoma sack"; "Digital stimulation or evacuation of the anorectum" was changed to "Digital evacuation or evacuation of the bowels" and "Frequency of fecal incontinence" was changed to "Frequency of fecal leakage along stoma sack". Like the original NBD score, the SMNBD thereby consists of ten items and total scores range from 0 to 47 . A score $\geq 14$ indicates severe bowel dysfunction.

Participants were asked which stoma-related problems they experienced in the last 4 weeks. To obtain more insight in diversion colitis, questions regarding experiencing loss of blood, moisture, and mucus in the last 3 months were asked. If so, questions with regard to frequency of rectal discharge and restrictions in daily life followed. Options were: "no restrictions at all", "minor restrictions", "moderate" or "major restrictions."

Satisfaction with choice for stoma formation was classified in five categories ("very dissatisfied", "dissatisfied", "neutral", "satisfied" or "very satisfied"). In addition, participants were asked if they would opt for a bowel stoma again and if they would advise someone with SCI to opt for a bowel stoma as well. Both items were scored on a five-point scale ("absolutely no", "no", "neutral", "yes" or "absolutely yes").

Satisfaction with timing of stoma formation was classified in five categories. Participants could choose if they experienced stoma formation as either 'far too early', "too early", "neutral" "too late" or "far too late".

Alterations in independence after stoma formation were scored on a 5-item scale ("much more dependent", "more dependent", "neutral or barely more (in)dependent", "more independent" or "much more independent".

Participants could score their satisfaction with regard to bowel management in the last four weeks either as "good", "adequate/acceptable", "bad" or "very bad". Alterations with regard to satisfaction with bowel management since stoma formation was scored on a 5-item scale ("much more deteriorated", "deteriorated", "neutral or barely deteriorated/ improved", "improved" or "much more improved").

Participants were asked to indicate alterations in satisfaction with their QOL on four domains (life as a whole, physical health, psychological health, social life) on a 5item scale ("much more deteriorated", "deteriorated", "neutral or barely deteriorated/improved", "improved" or "much more improved"). The four domains were taken from the International Spinal Cord Injury Data Sets-Quality of Life Basic Data set v2 [11, 12].

\section{Statistics}

All analyses were performed using the SPSS version 26.0. We performed descriptive statistics. Because of the small sample size, we did not perform inferential analyses.

\section{Results}

Data collection took place from February 2020 until March 26th 2020, when the COVID-19 crisis caused a premature closure of the questionnaire. In total, we sent 62 letters to participants known with SCI and a bowel stoma. Seventeen participants $(74 \%)$ completed the online questionnaire. Seven more participants responded to the open call for participation and completed the online questionnaire, but one of them needed to be excluded from analysis because he did not have a bowel stoma. The total number of participants is therefore 23. Their characteristics are displayed in Table 1. Stoma formation took place at thirteen different hospitals. Mean time since bowel stoma formation was 8.0 years (range 10 months-28.4 years, standard deviation 7.7 years). Twenty-two participants $(96 \%)$ received their bowel stoma $\geq 1$ year after onset of SCI. Most (20/23; 87\%) bowel stomas were single-looped colostomies.

Twenty-two participants $(96 \%)$ were satisfied with their choice for a bowel stoma and would choose to have one again (Table 2). Twenty-one participants (91\%) would advise someone else with SCI to opt for a bowel stoma and nineteen participants $(83 \%)$ reported to have become more independent after stoma formation. Nine participants (39\%) stated their bowel stoma was placed "too late". Another ten participants (44\%) answered their bowel stoma was placed "far too late". Not a single participant reported their stoma was placed "too early" or "far too early".

Sixteen participants (70\%) qualified their bowel management in the last 4 weeks as "good", six participants $(26 \%)$ as "adequate/acceptable" and one participant (4\%) as "bad". All but two $(21 / 23$; 91\%) participants reported improved satisfaction with their bowel management after stoma formation. One participant (4\%) stated satisfaction with bowel management barely improved, the other participant $(4 \%)$ reported deterioration in satisfaction with bowel management. However, both participants reported satisfaction with their choice for a bowel stoma and reported their bowel stoma was placed "too late". Seven participants (30\%) had moderate to severe SMNBD scores. Only one participant (4\%) with severe SMNBD score reported deterioration in satisfaction with bowel management after stoma formation.

Most participants reported improvements in all four items comparing QOL before and after bowel stoma formation: 20 participants $(87 \%)$ reported "improved" or "much more improved" satisfaction with life as a whole, 20 participants $(87 \%)$ reported "improved" or "much more improved" satisfaction with physical health, 19 participants $(83 \%)$ reported "improved" or "much more improved" satisfaction with psychological health and 19 participants $(83 \%)$ reported "improved" or "much more improved" satisfaction with social life. Only one participant (4\%) with 
Table 1 Characteristics of participants completing the online questionnaire.

\begin{tabular}{|c|c|}
\hline & $N=23$ \\
\hline Sex, male $(N, \%)$ & $11(48 \%)$ \\
\hline Age in years $($ mean $\pm S D)$ & $45.2 \pm 11.3$ \\
\hline Years since $\mathrm{SCI}($ mean $\pm \mathrm{SD})$ & $25.6 \pm 15.9$ \\
\hline Level of SCI, tetraplegia $(N, \%)$ & $7(30 \%)$ \\
\hline Level of SCI, paraplegia $(N, \%)$ & $16(70 \%)$ \\
\hline Completeness of SCI, complete $(N, \%)$ & $15(65 \%)$ \\
\hline Completeness of SCI, incomplete $(N, \%)$ & $8(35 \%)$ \\
\hline $\begin{array}{l}\text { Years since stoma formation after onset of SCI (mean } \pm \\
\text { SD) }\end{array}$ & $17.6 \pm 14.4$ \\
\hline Years since bowel stoma (mean $\pm \mathrm{SD})$ & $8.0 \pm 7.7$ \\
\hline \multicolumn{2}{|l|}{ Type of bowel stoma $(N, \%)$} \\
\hline Colostomy & $20(87 \%)$ \\
\hline Single looped & $18(78 \%)$ \\
\hline Double looped & $2(9 \%)$ \\
\hline Ileostomy & $3(13 \%)$ \\
\hline Single looped & $3(13 \%)$ \\
\hline Double looped & $0(0 \%)$ \\
\hline \multicolumn{2}{|l|}{ Tools used for evacuation prior to stoma formation $(N, \%)$} \\
\hline No intervention & $1(4 \%)$ \\
\hline Abdominal massage at least $15 \mathrm{~min}$ & $2(9 \%)$ \\
\hline Microlax klysma & $15(65 \%)$ \\
\hline Anal sphincter stimulation & $4(17 \%)$ \\
\hline Manual removal of feces & $7(30 \%)$ \\
\hline Transanal irrigation & $16(70 \%)$ \\
\hline \multicolumn{2}{|l|}{ Reasons for stoma formation $(N, \%)$} \\
\hline Abdominal pain & $3(15 \%)$ \\
\hline Obstipation & $7(30 \%)$ \\
\hline Fecal incontinence & $14(61 \%)$ \\
\hline Time spending for evacuation & $14(61 \%)$ \\
\hline Reducing dependency on caregivers & $2(9 \%)$ \\
\hline Other medical problmens ${ }^{\mathrm{a}}$ & $5(22 \%)$ \\
\hline Psychosocial issues $^{\mathrm{b}}$ & $3(12 \%)$ \\
\hline
\end{tabular}

$N$ number of participants; $S D$ standard deviation.

${ }^{a}$ Other medical problems: gut perforation, decubitus, malignancy (colon), perianal pain, insufficient effect after transanal irrigation.

${ }^{\mathrm{b}}$ Psychosocial issues: social isolation, freedom and uncertainty.

a very minor SMNBD score reported deterioration in one single QOL domain. The deterioration was in regard to satisfaction with their life as a whole.

Fourteen participants $(61 \%)$ did not report any stomarelated problem in the last month. Most commonly reported problem was excessive gas formation. Seven participants (30\%) reported symptoms of diversion colitis (loss of rectal blood, moisture, or mucus) in the last 3 months. Four out of these seven $(4 / 7 ; 57 \%)$ participants experienced loss of rectal blood, moisture or mucus at least once a week or
Table 2 Results online questionnaire.

\begin{tabular}{|c|c|}
\hline & $N=23$ \\
\hline \multicolumn{2}{|c|}{ Satisfaction with choice for bowel stoma $(N, \%)$} \\
\hline Very dissatisfied & $0(0 \%)$ \\
\hline Dissatisfied & $0(0 \%)$ \\
\hline Neutral & $1(4 \%)$ \\
\hline Satisfied & $8(35 \%)$ \\
\hline Very satisfied & $14(61 \%)$ \\
\hline \multicolumn{2}{|l|}{ Opting for a bowel stoma again $(N, \%)$} \\
\hline Absolutely no & $0(0 \%)$ \\
\hline No & $0(0 \%)$ \\
\hline Neutral & $1(4 \%)$ \\
\hline Yes & $8(35 \%)$ \\
\hline Absolutely yes & $14(61 \%)$ \\
\hline \multicolumn{2}{|c|}{ Advising someone else with SCI a bowel stoma $(N, \%)$} \\
\hline Absolutely no & $0(0 \%)$ \\
\hline No & $0(0 \%)$ \\
\hline Neutral & $2(9 \%)$ \\
\hline Yes & $9(39 \%)$ \\
\hline Absolutely yes & $12(52 \%)$ \\
\hline \multicolumn{2}{|c|}{ Alteration in independence after stoma formation $(N, \%)$} \\
\hline Much more dependent & $0(0 \%)$ \\
\hline More dependent & $0(0 \%)$ \\
\hline Neutral or barely (in)dependent & $4(17 \%)$ \\
\hline More independent & $9(39 \%)$ \\
\hline Much more independent & $10(44 \%)$ \\
\hline \multicolumn{2}{|c|}{ Experienced moment of getting a bowel stoma $(N, \%)$} \\
\hline Far too early & $0(0 \%)$ \\
\hline Too early & $0(0 \%)$ \\
\hline Neutral & $4(17 \%)$ \\
\hline Too late & $9(39 \%)$ \\
\hline Far too late & $10(44 \%)$ \\
\hline \multicolumn{2}{|c|}{ Satisfaction with bowel management in the last 4 weeks $(N, \%)$} \\
\hline Very bad & $0(0 \%)$ \\
\hline Bad & $1(4 \%)$ \\
\hline Adequate/acceptable & $6(26 \%)$ \\
\hline Good & $16(70 \%)$ \\
\hline \multicolumn{2}{|c|}{ Satisfaction with bowel management since stoma formation $(N, \%)$} \\
\hline Much more deteriorated & $0(0 \%)$ \\
\hline Deteriorated & $1(4 \%)$ \\
\hline Neutral or barely deteriorated/improved & $1(4 \%)$ \\
\hline Improved & $7(30 \%)$ \\
\hline Much more improved & $14(61 \%)$ \\
\hline SMNBD score $($ mean $\pm \mathrm{SD})$ & $6.6 \pm 5.6$ \\
\hline \multicolumn{2}{|l|}{ SMNBD categories $(N, \%)$} \\
\hline Very minor SMNBD (score $0-6$ ) & $15(65 \%)$ \\
\hline Minor SMNBD (score 7-9) & $1(4 \%)$ \\
\hline Moderate SMNBD (score 10-13) & $3(13 \%)$ \\
\hline Severe SMNBD (score $\geq 14$ ) & $4(17 \%)$ \\
\hline
\end{tabular}

Alteration in satisfaction with QOL since stoma formation with regard to life as a whole $(N, \%)$

Much more deteriorated $\quad 0(0 \%)$

Deteriorated $1(4 \%)$

Neutral or barely deteriorated/improved $2(9 \%)$

Improved $8(35 \%)$

Much more improved $12(52 \%)$

Alteration in satisfaction with QOL since stoma formation with regard to physical health $(N, \%)$

Much more deteriorated $0(0 \%)$ 
Table 2 (continued)

\begin{tabular}{|c|c|}
\hline & $N=23$ \\
\hline Deteriorated & $0(0 \%)$ \\
\hline Neutral or barely deteriorated/improved & $3(13 \%)$ \\
\hline Improved & $9(39 \%)$ \\
\hline Much more improved & $11(48 \%)$ \\
\hline \multicolumn{2}{|c|}{$\begin{array}{l}\text { Alteration in satisfaction with QOL since stoma formation with regard to } \\
\text { psychological health }(N, \%)\end{array}$} \\
\hline Much more deteriorated & $0(0 \%)$ \\
\hline Deteriorated & $0(0 \%)$ \\
\hline Neutral or barely deteriorated/improved & $4(17 \%)$ \\
\hline Improved & $7(30 \%)$ \\
\hline Much more improved & $12(52 \%)$ \\
\hline \multicolumn{2}{|c|}{$\begin{array}{l}\text { Alteration in satisfaction with QOL since stoma formation with regard to socia } \\
\text { life }(N, \%)\end{array}$} \\
\hline Much more deteriorated & $0(0 \%)$ \\
\hline Deteriorated & $0(0 \%)$ \\
\hline Neutral or barely deteriorated/improved & $4(17 \%)$ \\
\hline Improved & $8(35 \%)$ \\
\hline Much more improved & $11(48 \%)$ \\
\hline \multicolumn{2}{|c|}{ Reported side effects of bowel stoma in the last month $(N, \%)$} \\
\hline Skin irritation around stoma attachment & $3(13 \%)$ \\
\hline Fecal leakage & $4(17 \%)$ \\
\hline Excessive gas formation & $5(22 \%)$ \\
\hline Complains due to constipation & $1(4 \%)$ \\
\hline Abdominal pain (cramps) & $1(4 \%)$ \\
\hline No problems with stoma & $14(61 \%)$ \\
\hline
\end{tabular}

$N$ number of participants, $S M N B D$ stoma-modified neurogenic bowel dysfunction.

more. Two out of these seven $(2 / 7 ; 29 \%)$ participants stated this had a moderate impact on their daily activities.

\section{Discussion}

In our present study, we found 22 participants (96\%) were satisfied with their bowel stoma, even in the presence of severe SMNBD or stoma related side effects. Our results with regard to satisfaction with bowel stoma in individuals with SCI are in line with results from other studies. Rosito et al. reported 27 participants $(27 / 27 ; 100 \%)$ were satisfied with their colostomy. However, three participants (11\%) wanted their colostomy reversed, which was not further specified [13]. In this study, the mean time since formation was 8.0 years (range 1.5-28 years). Bølling Hansen et al. reported 17 participants $(17 / 18 ; 94 \%)$ were satisfied with their decision to choose for a colostomy [14]. In this study, the mean time since stoma formation was 6.9 years (range 6 months -20 years). The one participant who wanted his colostomy reversed had initially received his colostomy due to an infection and did not have problems with bowel management prior to stoma surgery.

Our study is one of the few studies addressing individuals directly about whether they thought their stoma was placed too early or too late. Our finding that almost all of the participants stated their bowel stoma was placed too late is in line with other studies. The study from Rosito et al. reported 19 participants $(19 / 27 ; 70 \%)$ wanted to have their colostomy earlier [13]. Branagan et al. reported 25 participants $(25 / 30 ; 83 \%)$ wanted to have been offered a stoma earlier [7]. In this study, the mean time from onset of SCI to stoma formation was 17 years (range $0-36.25$ years). Coggrave et al. stated 49 participants $(49 / 92 ; 53 \%)$ with SCI found their bowel stoma was not placed at the right time [15]. Mean time since stoma formation after onset of SCI was 19 years (range 5 months-28 years). None of the participants stated stoma formation was too early. The question which comes to mind then is the following: in what time frame do we have to think about when individuals with SCI quote they wanted to have a bowel stoma earlier? The formation of a bowel stoma after several years of problematic, time spending stool evacuations can be a life changer for individuals with SCI. Maybe, if individuals with SCI would get a bowel stoma "earlier", they would not score their self-reported improvements regarding QOL the way participants did in our study. This could be due to the fact because they simply did not have the frame of reference and time to overthink their personal situation regarding bowel management compared to individuals who received a bowel stoma after many years. The results of the above-described studies, together with the results of our study, imply that the formation of a bowel stoma should be offered earlier, or at least be considered earlier in individuals with SCI.

In our study, seven participants $(30 \%)$ reported complaints from diversion colitis. Two out of these seven participants $(2 / 7 ; 29 \%)$ stated this had a moderate influence on their daily life. One of our participants reported that his colon distal to his colostomy was surgically removed due to rectal moisture. This individual recommended everyone with the same problem this surgical operation. Other studies reported higher percentages of rectal discharge in individuals with SCI. Boucher et al. retrospectively studied 72 participants with SCI with either early colostomy formation (in the first few months after onset SCI; 20 participants) or late colostomy formation (after at least one year; 52 participants) [16]. Early complication rates did not differ between both groups. However, participants with early colostomy formation experienced more rectal discharge compared to the late colostomy group ( $40 \%$ versus $11.5 \%$ ). In this study, there was one participant from the late colostomy group who was dissatisfied with his bowel stoma who eventually underwent colostomy reversal. The study from Branagan et al. reported that fourteen participants $(14 / 32 ; 44 \%)$ with SCI and bowel stoma had leakage of mucus, blood or pus per rectum [7]. Coggrave et al. reported 42 participants $(42 / 92 ; 46 \%)$ to have rectal mucous discharge [15]. The aforementioned studies, however, did not report whether rectal leakage had a negative 
influence on daily life. Based on our small study size, more research is warranted to investigate this topic in this specific pool of patients.

To the best of our knowledge, this study is the first study to address alterations in four validated domains with regard to self-reported QOL after stoma formation in individuals with SCI. Other studies addressing QOL after stoma formation in individuals with SCI reported similar outcomes. Branagan et al. reported 25 participants $(25 / 31 ; 81 \%)$ with SCI experienced improvements after stoma formation regarding QOL. There was one participant who experienced deterioration in this study [7]. Rosito et al. reported 23 participants $(23 / 27 ; 85 \%)$ felt an increase in QOL after colostomy formation [10]. Cooper et al. reported 20 participants $(20 / 26 ; 77 \%)$ to have improvements of their healthrelated QOL [6]. The other six participants $(23 \%)$ stated their QOL was not changed.

The first main limitation of the current study is its small sample size, partly due to the early closure of the study due to the COVID-19 outbreak. Previous studies also concerned small samples, as the application of bowel stoma after SCI is still limited. Nevertheless, this small sample size limits the generalizability of our study results and made it impossible to explore differences between the ileostomy and colostomy groups. The second main limitation is the use of a modified version of the NBD questionnaire. The SMNBD questionnaire was based on the validated version of NBD score. Our modified version has not been validated yet and scores on this SMNBD and the cutoff point to indicate severe NBD cannot be considered equivalent to the original NBD score without further validation. Therefore, we recommend new studies to elaborate a standardized, validated questionnaire for daily practise to evaluate NBD in individuals with SCI and bowel stoma. For example, adopting a Delphi process like the method adopted for the creation of the MENTOR tool by Emmanuel et al. [17]. The last limitation of our study concerns the quality of our data. We did not have access to medical files and therefore had to rely on self-reported data.

\section{Conclusion}

Our study suggests the majority of individuals with SCI and bowel stoma in retrospect wanted to have a bowel stoma earlier. Besides, the majority of individuals reported improvements in four domains regarding QOL after stoma formation. Early, extensive conversations about bowel management at the inpatient and outpatient rehabilitation after onset of SCI should take place. Individuals should be informed about the option to choose for stoma formation, which is a safe and effective option after SCI. Collaboration should take place in a multidisciplinary setting with rehabilitation physicians, surgeons specialized in operating neurogenic bowels, and specialist stoma nurses.

\section{Data archiving}

The online questionnaire and data supporting the findings of this study are available upon reasonable request.

Acknowledgements I would like to thank all study participants because without them this work would not be possible.

\section{Compliance with ethical standards}

Conflict of interest The authors declare that they have no conflict of interest.

Publisher's note Springer Nature remains neutral with regard to jurisdictional claims in published maps and institutional affiliations.

\section{References}

1. Adriaansen JJ, van Asbeck FW, van Kuppevelt D, Snoek GJ, Post MW. Outcomes of neurogenic bowel management in individuals living with a spinal cord injury for at least 10 years. Arch Phys Med Rehabil. 2015;96:905-12.

2. Adriaansen JJ, Ruijs LE, van Koppenhagen CF, van Asbeck FW, Snoek GJ, van Kuppevelt D, et al. Secondary health conditions and quality of life in persons living with spinal cord injury for at least ten years. J Rehabil Med. 2016;48:853-60.

3. Emmanuel A. Neurogenic bowel dysfunction. F1000 Res. 2019;8:1800

4. Coggrave M, Norton C, Cody JD. Management of faecal incontinence and constipation in adults with central neurological diseases. Cochrane Datab Syst Rev. 2014:CD002115. https://doi.org/ 10.1002/14651858.CD002115.pub5.

5. Emmanuel A. Rehabilitation in practice: managing neurogenic bowel dysfunction. Clin Rehabil. 2010;24:483-8.

6. Cooper EA, Bonne Lee B, Muhlmann M. Outcomes following stoma formation in patients with spinal cord injury. Color Dis. 2019;21:1415-20.

7. Branagan G, Tromans A, Finnis D. Effect of stoma formation on bowel care and quality of life in patients with spinal cord injury. Spinal Cord. 2003;41:680-3.

8. Shabbir J, Britton DC. Stoma complications: a literature overview. Color Dis. 2010;12:958-64.

9. Tominaga K, Kamimura K, Takahashi K, Yokoyama J, Yamagiwa S, Terai S. Diversion colitis and pouchitis: a mini-review. World J Gastroenterol. 2018;24:1734-47.

10. Krogh K, Christensen P, Sabroe S, Laurberg S. Neurogenic bowel dysfunction score. Spinal Cord. 2006;44:625-31.

11. Charlifue S, Post MW, Biering-Sørensen F, Catz A, Dijkers M, Geyh $\mathrm{S}$, et al. International spinal cord injury quality of life basic data set. Spinal Cord. 2012;50:672-5.

12. Post MW, Forchheimer MB, Charlifue S, D'Andrea Greve JM, New PW, Tate DG. Reproducibility of the international spinal cord injury quality of life basic data set: an international psychometric study. Spinal Cord. 2019;57:992-8. 
13. Rosito O, Nino-Murcia M, Wolfe VA, Kiratli BJPI. The effects of colostomy on the quality of life in patients with spinal cord injury: a retrospective analysis. J Spinal Cord Med. 2002;25:174-83.

14. Bølling Hansen R, Staun M, Kalhauge A, Langholz E, BieringSørensen F. Bowel function and quality of life after colostomy in individuals with spinal cord injury. J Spinal Cord Med. 2016;39:281-9.

15. Coggrave MJ, Ingram RM, Gardner BP, Norton CS. The impact of stoma for bowel management after spinal cord injury. Spinal Cord. 2012;50:848-52.
16. Boucher M, Dukes S, Bryan S, Branagan G. Early colostomy formation can improve independence following spinal cord injury and increase acceptability of bowel management. Top Spinal Cord Inj Rehabil. 2019;25:23-30.

17. Emmanuel A, Krogh K, Kirshblum S, Christensen P, Spinelli M, van Kuppevelt D, et al. Creation and validation of a new tool for the monitoring efficacy of neurogenic bowel dysfunction treatment on response: the MENTOR tool. Spinal Cord. 2020;58:795-802. 